\title{
Quantification of happy emotion: Proportionality relationship to gain/loss
}

\author{
Nicoladie D. Tam \\ Department of Biological Sciences, University of North Texas, Denton, TX 76206, USA
}

Email address:

nicoladie.tam@unt.edu

\section{To cite this article:}

Nicoladie D. Tam. Quantification of Happy Emotion: Proportionality Relationship to Gain/Loss. Psychology and Behavioral Sciences. Vol. 3, No. 2, 2014, pp. 60-67. doi: 10.11648/j.pbs.20140302.15

\begin{abstract}
Toward understanding the role of emotion played in cognitive processing, an emotional model has been proposed to quantify the computation involved in assessing the disparity between the expected and the actual outcomes. This study provides the experimental evidence to validate the above emotional model. In this model, emotion serves as an internal feedback to assess the disparity between the internal predicted outcomes and the actual (external) outcomes in reality. It predicts that emotion provides a feedback to reduce the discrepancy between the expected (subjective) reality and actual (objective) reality. The hypothesis for this model is that the intensity of emotional response is proportional to the disparity between the expected outcome and the actual outcome (i.e., gain/loss magnitude). Happiness is an emotional feedback that indicates the congruency between the predicted and actual outcomes. In order to validate this theoretical model of emotion, the classical Ultimatum Game (UG) is used as an experimental paradigm to elicit self-generated (endogenous) emotions in response to a monetary offer, so that the emotional responses with respect to the perceived monetary gain/loss can be assessed by the stimulus-response function. The results showed that the self-reported happiness intensity is directly proportional to the magnitude of the desirable monetary gain. An empirically derived emotion stimulus-response function is shown to quantify the specific emotional biases graphically by the emotional-disparity graph. The results validated the hypothesis that the intensity of self-reported happy emotion is directly proportional to the monetary gain. The analysis also showed that the happy emotional sensitivity is also changed by the perception of fairness (whether the offer is fair or unfair), which can be represented graphically by the emotional-disparity graph.
\end{abstract}

Keywords: Emotional Model, Happy, Fairness, Gain, Ultimatum Game, Decision Making, Error Minimization

\section{Introduction}

Emotion is a well-recognized phenomenon that provides important survival functions for animals and humans. Toward this goal to examine the functional role of emotions in survival, a neurobiological model had been developed to explore the computations involved in emotional processing $[1,2]$. Using a minimal assumption approach, it can be shown that an objective model of emotion can be quantified using basic principles of survival $[1,2]$.

Rather than using a traditional model of emotion, this model proposed that emotion is evolved to provide an internal feedback to an organism to assess the accuracy of its internal predictions of the outcomes in the real world. If the internally generated model of the world by its brain is an accurate model, then the survivability of the organism will increase. If the internally generated prediction is inaccurate, then the survivability will decrease in the real world. The internal model of the external world is essentially the belief system of the organism, in which it predicts how to interact with the real world accurately for survival. The disparity between the internal model and the external world provides a self-derived measure to assess the accuracy of the internal model. The bigger the disparity, the greater the inaccuracy is assessed. Thus, an organism can correct itself by this neurobiological self-assessment mechanism automatically (as an autonomic response) without necessary any conscious control. This discrepancy signal is essentially the emotional feedback for correcting any inaccuracy of its internal model prediction with respect to the sensory (perceptual) or execution (motor) errors.

The initial step in this self-discovery of inaccuracy of the internal model is the detection of the existence of error, based on the disparity between the model prediction and the 
actuality. Once the source of error has been self-discovered, then steps can be taken to correct the error, so that congruency between the internal prediction and the actual outcome can be achieved, resulting in an increase in survivability of the organism. The congruency is represented by the happy emotion, while incongruency is represented by the unhappy emotion. This serves as an internal feedback mechanism for assessing errors, in which error-detection and error-correction can be achieved by the emotional processing circuitry. Once the errors are reduced, the state of happiness serves as an internal feedback to the organism to signify the sense of content, in which no further error-corrections are needed. Thus, it reaches a state of internal equilibrium emotionally, once the incongruencies are resolved. Emotional resolution is an internal process in which these errors and incongruencies are resolved by the brain.

This quantitative computational model of emotion has been validated experimentally in human subjects, which showed that the intensity of emotion is proportional to the disparity between the expected and actual outcomes. Specifically, it showed that the anger emotion is directly proportional to the magnitude of the loss (disparity between the expected and actual outcomes) [3]. In this study, we will show that the happy emotion is proportional to the gain, opposite to the inverse proportional relationship of anger emotion to the gain.

\subsection{The Unified Theoretical Model of Emotion}

The theoretical model of emotion is based on two emotion models combined together. The EMOTION-I model proposes that emotion is evolved as a mechanism for increasing survival by assessing the contextual information of the environment to produce a more appropriate response [1]. The EMOTION-II model proposes that emotion is a feedback signal for increasing survival by assessing the accuracy of the internal (brain) model of the external world [2]. That is, the probability of survival of an animal is dependent on the appropriateness of the response (as proposed by the EMOTION-I model) [1] and the accuracy of the prediction of the interactions with the external world (as proposed by the EMOTION-II model) [2].

A unified model is proposed by combining the above two models, where emotion is a feedback for an animal to assess the discrepancy between the internal reality (internal model prediction) and the external reality (actuality in the external world). The likelihood of survival will increase if the internal (brain) model accurately predicts the interactions with the external world (without any errors). The likelihood of survival will decrease if the predictions are inaccurate. Thus, emotions serve as the vital feedback signals for an animal to detect, identify and correct any errors that may exist. When errors are detected, the state of unhappiness serves as the feedback for an animal to identify and correct such errors. Once these errors are corrected, an accurate prediction of the external world will produce congruency between the expected and actual outcomes, reaching the state of happiness.

Intuitively, emotions are assessments of the difference between what one wants and what one gets. An animal is happy, if it gets what it wants (given that what it wants is also desirable in survival). If it does not get what it wants, it may be unhappy. This is consistent with the tendency that we often ask, "what is wrong?" when we see someone unhappy, so as to help him/her to reduce the discrepancy between what he/she expects and what he/she gets.

Computationally, the emotional model predicts that the emotional response is an internal feedback signal to reduce the error (disparity) between the internal model of the world (expected outcome) and the external world (actual outcomes. Thus, resolving an emotion is essentially an error-minimization process to reduce the disparity between the "wants" and "gets," so that congruency between the expected and actual outcomes can be achieved [4]. Happiness is the internal emotional feedback to indicate the congruency between the expected and actual outcomes, while unhappiness is the internal feedback to indicate that there is a discrepancy (incongruency) between the expected and actual outcomes.

In order to provide a quantitative assessment of these wants and gets, the brain has to compute and estimate the "gains" and "losses." The gain/loss signals provide the quantifiable variables needed for computing the emotional response with respect to the expectancy. If such gains were desirable, the emotional feedback would be a happy emotional response. This happy emotion would provide the necessary feedback as an indicator that the brain's prediction is congruent with the external world, which means that the brain's prediction (belief system) is accurate. Otherwise, unhappy emotion is triggered to indicate incongruency, i.e., some errors must have existed.

\subsection{Stimuli for Eliciting Emotional Responses}

Although emotions have been studied extensively in relation to animal's behavior, due to the subjective nature of emotions, it is often difficult to quantify objectively. Traditionally, emotions are often quantified and elicited experimentally using facial expressions or emotional words as the stimuli [5]. But the emotional ratings elicited by facial expressions as stimuli are often dependent on many other factors, such as visual field [6] and mental disorders [7]. The mental disorders affected by emotions include unipolar depression [8], anxiety disorder, [9] and schizophrenia [10]. Furthermore, the ability to perceive emotions in others is also affected by the ability to activate similar emotion in oneself, particularly in disorders such as alexithymia [11, 12]. Alternatively, emotional words are also commonly used as stimuli to elicit emotional responses [13, 14] or an emotional movie scene. Yet these stimuli (facial expressions, emotional words or movies) are non-quantifiable numeric stimuli to elicit emotional response. Since the goal of this study is to establish the stimulus-response function for emotion, it requires quantification of both the stimulus and response with numerical measures. 


\subsection{Experimental Paradigm to Validate the Theoretical Model of Emotion}

Toward this goal to quantify both the emotional stimulus and response, this study combined the theoretical emotion model $[1,2]$ and the experimental paradigm of ultimatum game (UG) [15] to establish a stimulus-response function for emotion quantitatively.

In order to confirm whether humans respond emotionally with respect to the gain/loss signals, we use the classical ultimatum game (UG) paradigm. UG is widely used in behavioral economics in the past 60 years to assess emotions and decision-making responses in neuroscience, psychology, social science, economics and mathematical psychology [15-18] with hundreds of studies published to examine the decision-making process in humans. It is also used to examine the emotional response in relation to the decision-making process [19-28].

UG is a simple split-the-money game, in which an amount of money (such as \$10) is divided between two persons (a proposer and a responder). If the responder accepts the offer, then they both keep the money. If the responder rejects the offer, both parties lose the money. Although most studies use the UG paradigm to examine the decision-making process [20,27-31], we use this paradigm to elicit self-generated (endogenous) emotions in relation to the gain/loss disparity. That is, we employed a quantitative and objective stimulus (monetary disparity) to elicit an emotional response rather than using subjective (non-quantitative) stimuli, such as facial expression or emotional words, to elicit an emotional response.

It has been shown that emotions do interact with decisions, especially in UG paradigm [19-28], which provided the validate justifications for using UG paradigm to elicit emotions. Thus, the UG paradigm is used as the experimental paradigm to elicit emotions in humans using a numerical quantifiable variable - the monetary offer-ratio between the proposer and responder. This allows the elicitation of emotional response without resorting to use subjective facial expressions or emotional words.

\section{Methods}

Healthy human subjects were recruited in the UG experiment, where the subjects played as the "responder" to accept or reject the monetary proposal presented to them on a computer screen. We did not indicate whether the "proposer" is human or computer because it has been shown that knowledge of whether it is proposed by a human or a computer could bias the perception and response of the subjects in $U G$, if such hints were made [28, 29]. Nine randomized offers were proposed to the subjects ranging from stingy $\$ 1: \$ 9$ offers to generous $\$ 9$ : $\$ 1$ offers. The trials were randomized so that the subject cannot guess at the amount of money being offered. Self-reported emotional ratings (+5 to -5$)$ were recorded immediately after the participants accepted (or rejected) each offer in the one-shot trial experiment (without repeating the same randomized offer-ratios). The emotional ratings included happy, sad, angry and jealous emotions (using the same +5 to -5 Likert scale [32]). The subjects were also asked to rate other attributes — including whether they won the trial; whether the offer was fair; how important fairness is; how important money is; and how important winning is to them — using the same +5 to -5 scale. The other self-reported ratings served, in part, as distracters to reduce the likelihood of the subjects to skew their responses according to what they expected from the experimenter.

As with other UG experiments [22, 28, 30, 31, 33-45], the experiments were designed without asking the subjects explicitly why they opted to accept/reject the offer; why they felt the way they did emotionally; what their perceived gains/losses were; and whether money or fairness was what they wanted in the experiment. Otherwise asking such questions could skew their responses. This paper focuses on analyzing the happy emotion, while the analysis of other emotions were provided elsewhere $[4,41,46]$; specifically, it has already been confirmed that an inverse proportional relationship exists between the intensity of anger and the size of gain/loss signals [41]. The experimental protocol was approved by the Institutional Review Board.

\section{Results}

A total of 425 voluntary subjects were included in this study (age ranging from 18 to 80 , median $=21$; mean $=$ 22.3; $\mathrm{SD}=4.7$ ). Fig. 1 shows the self-reported rating of the happiness emotion for the entire sampled population, independent of whether they accepted or rejected the offer. (Note the responses were sorted in the graphs according to the ascending order of offer-ratios from the original randomized orders.) It shows a direct proportional relationship between self-reported happiness rating and the monetary offer-ratio (Fig. 1), with a high regression coefficient $\left(r=0.939, r^{2}=0.881\right)$. This shows a strong correlation between the emotional intensity and the offer-ratio, as predicted by the theoretical emotional model.

Note that the proportional relationship does not pass through the origin of the axes. That is, the neutral emotional threshold (happiness rating of 0 ) is neither located at the axis-origin nor at the \$5:\$5 even-split (most equitable fair-share offer). The crossover threshold point (for neutral emotion) is at $\$ 2: \$ 8$ offer-ratio instead, similar to the crossover threshold for rejection in other UG studies $[3,47]$. This means it takes a minimum gain of a $\$ 2$ offer to make them happy; anything less is unhappy.

The shift in the threshold for neutral emotion quantifies the emotional bias graphically. If fairness (or equity) were the criterion for what they want, then the $\$ 5: \$ 5$ even-split would be the most equitable and fair offer; anything less would be inequitable and unfair, and above that would be hyper-fair and hyper-equitable [48]. When the emotional threshold crossover point is located at $\$ 2: \$ 8$ offer-ratio (Fig. 1), it suggests that subjects were rather happy just 
getting some money, and the offer did not need to be equal or fair (absolutely even) in order for them to be happy. Thus, this stimulus-response graph quantified the relationship between the emotional response and the offer-ratio, which showed a proportionality relationship between them. The more favorable the monetary offer, the higher the self-reported happy emotional intensity is.

Although the stimulus-response function approximates a direct-proportional function, there are some anomalies in the fitted curve. The emotional response to the $\$ 5: \$ 5$ even-split offer (representing the fairest offer objectively) deviates from the fitted regression line. This suggests that the human subjects reported happier to the $\$ 5: \$ 5$ fair-offer than the $\$ 6: \$ 4$ generous-offer. Thus, this reveals that the emotional response is not only related to the expected gain, but can also be biased by the perception of objective fairness. That is, even though the $\$ 6: \$ 4$ generous-offer is more than fair subjectively (relative to self), the intensity of happiness is not as high as the objectively $\$ 5: \$ 5$ fair-offer (relative to both parties - self and other). Thus, this shows that the sense of objective fairness can override the sense of subjective selfishness in the hyper-fair (more than fair) offer. The emotional response can be higher for objective fairness than subjective fairness.

In order to assess the difference in emotional response to the relativity of fairness (i.e., relative to self vs. relative to other), Fig. 2 shows the same graph as Fig. 1, except that it is fitted with two separate regression lines instead of one. The regression lines are fitted according to the relative fairness - one regression line is fitted according to the unfavorable offer-ratio (i.e., unfair offers), while the other regression line is fitted according to the favorable offer-ratio (i.e., hyper-fair offers).

The stimulus-response function in Fig. 2 reveals that the emotional intensity is proportional to the offer-ratio for both unfair offers $\left(r=0.953, r^{2}=0.909\right)$ (left-half of Fig. 2) and hyper-fair offers $\left(r=0.929, r^{2}=0.863\right)$, except that the slopes are different. The slope for the unfair offers (slope $=0.623$ ) (left-half of Fig. 2) is twice as steep as the slope for the hyper-fair offers (slope $=0.311$ ). This suggests that the emotional responses are two times more sensitive to fairness, when the offers are unfair, than the offers that are hyper-fair. In other words, the human subjects reported that their emotional responses are highly sensitive to the unfair offers (with a steeper slope), while their emotional responses are much less sensitive to the hyper-fair offers (with a shallower slope).

Note that the slope for the overall graph $($ slope $=0.637)$ (Fig. 1) is similar to the slope for the unfair offers (slope $=0.623$ ) (left-half of Fig. 2). This suggests that the emotional sensitivity did not change with unfair offers. Rather, the emotional sensitivity decreased by half (slope $=0.311$ ) for the hyper-fair offers (right-half of Fig. 2). This suggests the desensitization effect by hyper-fairness, as revealed by a decrease in the slope of the stimulus-response function.

This suggests that when the offers are more than fair, the human subjects responded with less emotional sensitivity to happiness than unfair offers. On the other hand, the emotional sensitivity to unfair offers remained unchanged compared to the overall emotional responses, with one exception (comparing Fig. 1 and left-half of Fig. 2). The exception is that when the offer is an objective fair-share offer at the absolute equity $\$ 5: \$ 5$ offer-ratio, then the happy emotion is greatly enhanced (elated).

More interestingly, the emotional baselines are shifted according to the fairness perception, as revealed by the $y$-intercepts of stimulus-response functions. The $y$-intercept for the unfair offers is -1.512 (left-half of Fig. 2, from the regression line: $y=0.623 x-1.512$ ), while the $y$-intercept for the hyper-fair offers is +1.071 (right-half of Fig. 2). This shows that the emotional baseline for the unfair offers is negative, while the emotional baseline for the hyper-fair offers is positive. This suggests that the emotional baseline can be shifted up from a negative emotion to a positive emotion by the fairness perception, with a happier emotional baseline for the hyper-fair offers than the unfair offers. Nonetheless, the emotional intensity for absolute equity $\$ 5: \$ 5$ (objective fairness) is higher than the baseline emotion for hyper-fairness.

Note also that the $y$-intercept $(-1.278)$ for the overall graph (Fig. 1) is similar to the $y$-intercept $(-1.512)$ for the unfair offers (left-half of Fig. 2). This shows that the emotional baseline remains unchanged for the overall emotional response and for the unfair response. This phenomenon is similar to how the emotional sensitivity remains unchanged for the unfair offer, but it changed for the hyper-fair offers, as discussed earlier. On the other hand, the emotional baseline $(y$-intercept $=+1.071)$ is shifted to the positive emotion, while the emotional sensitivity decreased by half (from the slope $=0.623$ to slope $=0.311$ ) for the hyper-fair offers (Fig. 2).

Thus, this analysis quantitatively identifies how the emotional baseline ( $y$-intercept) is shifted (up or down) by the fairness perception, and how the emotional sensitivity (slope) is changed (shallower or steeper) by the fairness perception, as revealed graphically by the happy emotion stimulus-response functions.

\section{Discussion}

The data revealed the quantitative proportional relationship between emotional intensity and the disparity of gain/loss. It is consistent with the hypothesis that the more we gain, the happier we are; when the gain is desirable and favorable (rather than undesirable or unfavorable). Conversely, the more we lose, the less happy we are. This direct proportional relationship provided a quantitative measure for assessing how emotional responses can be biased. That is, the stimulus-response function of the emotional-disparity graph approximates a piecewise linear function, which can be shifted up or down, or tilted by changing the slope. 


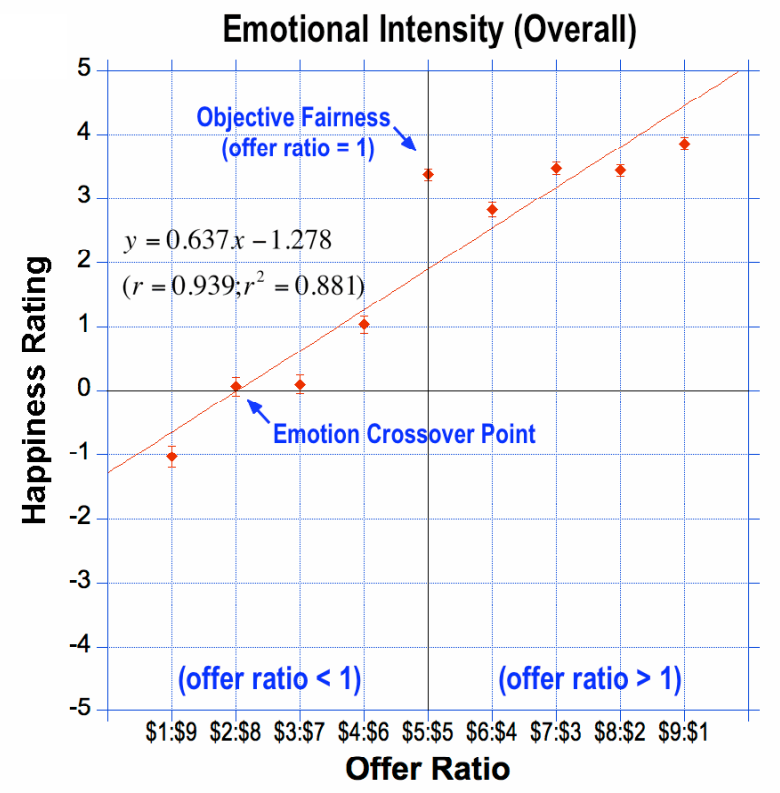

Figure 1. Average response of entire population in our sample $(n=425)$ for self-reported emotional rating of happiness with respect to monetary offer-ratios. The fitted regression line shows a linear proportional relationship between the emotional intensity and the offer-ratios. Error bars represent standard errors of means (SEMs).

More interestingly, the above quantitative analysis also revealed some anomalies to the above proportionality relationship. First, it revealed that the human subjects can identify the difference between objective and subjective fairness, by reporting a higher happiness rating for an objective $\$ 5: \$ 5$ even-split fair-offer than the subjective $\$ 6$ : \$4 hyper-fair offer. Thus, it suggests that the emotional response is not only dependent on the magnitude of gain, but also on the relative fairness perception. The sense of objective fairness in equality is happier than the sense of subjective fairness with a bigger gain.

Second, it also revealed that the sensitivity of emotional responses is also biased by the degree of fairness. The emotional response is more sensitive to unfair offers than hyper-fair offers. That is, when the offers are unfair, it will elicit a bigger emotional response than when the offers are hyper-fair. This suggests that when a person receives a hyper-fair offer, it elicits a much lesser emotional response than when a person receives an unfair offer. Thus, this quantifies that humans pay more attention (hyper-sensitive) to unfair offers than hyper-fair offers. It is less important when the person has already gained more than its share. Thus, the above analysis quantified how emotional response is biased by the perception of fairness (unfair offers compared to the hyper-fair). This is represented by the change in the slope of the stimulus-response function. The slope is steeper for the unfair offers than the hyper-fair offers.

To further our analysis, let us assume both money and fairness are the desirable goals wanted by the subjects. Let

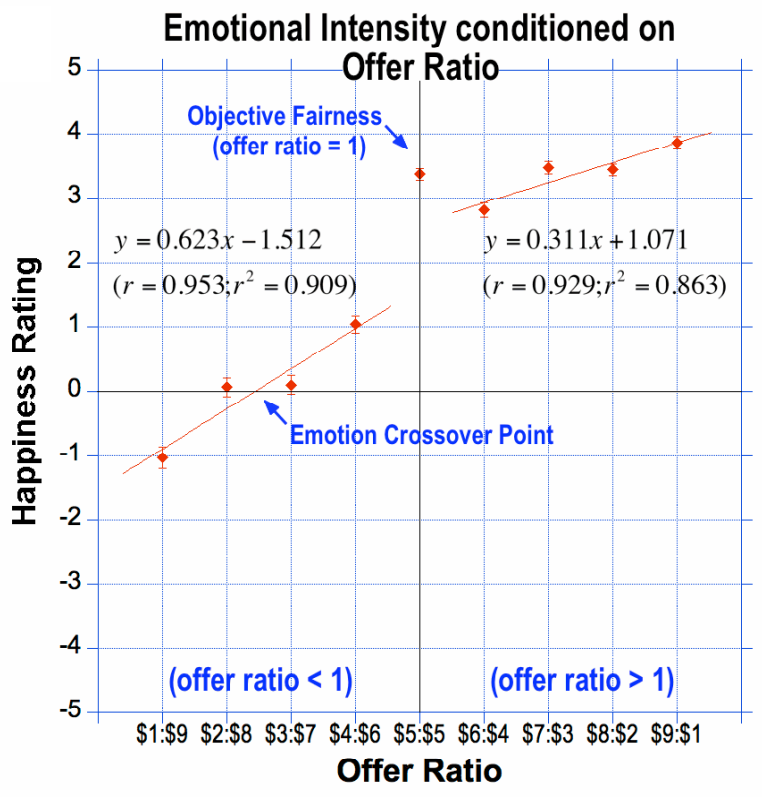

Figure 2. Same graph as in Fig. 1 except that the regression lines are fitted according to the unfair offers (left-half) and the hyper-fair offers (right-half). This shows that the stimulus-response function is steeper for the unfair offers than the hyper-fair offers. This represents a change in emotional sensitivity according to the perception of unfair vs. hyper-fair.

us also assume fairness is centered on the absolute equity $\$ 5: \$ 5$ ratio (fair share) in social transactions $[27,28,35$, $36]$, even though it has been shown that the subjects often biased the fairness threshold to an inequitable offer-ratio of $\$ 2: \$ 8$ instead of $\$ 5: \$ 5[41,49]$. Then the UG creates the classical dilemma for unfair offers - they can only get one (either fairness or money), but not both.

Our analysis shows that human subjects displayed a sense of lenience toward unfair offers, responding to the unfair (inequitable) offers as happy (such as $\$ 3: \$ 7$ ) rather than unhappy, unless the offer is less than the $\$ 2: \$ 8$ offer-ratio. That is, they shifted their threshold of happiness from the absolute equity $\$ 5: \$ 5$ (even-split) offer-ratio to a rather unfair offer of $\$ 2: \$ 8$. This is quantified by the threshold crossover point at the $\$ 2: \$ 8$ offer-ratio instead of the $\$ 5: \$ 5$ offer-ratio (see Figs 1 and 2). It shifts the stimulus-response function to the left without altering the proportional relationship between the emotional response and the offer-ratio. This is consistent with the findings in other UG studies that emotional responses are related to unfairness [35, 36], anger and spite [31, 39], or altruistic punishment $[47,50]$ rather than being irrational.

These findings may also shed light on the emotional processing neural circuitry for assessing the amount of gain/loss. Satiation could devalue the size of the gains in the emotional feedback. This devaluing effect in satiation is a well-known innate response in animals when they have gotten (satisfied with) what they wanted. For instance, when monkeys were completely satisfied with consuming a large quantity of peanuts, they would devalue the gains 
(peanut rewards) by choosing to eat raisins instead of peanuts, if given the choice [51].

The amygdala is known to be one of the crucial brain structures in producing this devaluing effect [52], which updates the re-valued information to the orbitofrontal cortex (OFC) [53] for final decision in choice preference [54]. This suggests that the amygdala (a known brain structure for processing emotional response) is also involved in processing gain/loss assessment by devaluing the gain signal.

The gain/loss assessment neural pathways also include the emotional circuitry in the striatum, ventromedial prefrontal cortex (PFC), ventral anterior cingulate cortex (ACC) and medial OFC, which increase the neural activity for gains, but decrease the activity for losses [55]. Taken together, our result is consistent with other UG studies that subjects reported a higher level of positive affect (i.e. happier), when subjects received fair offers than when they received unfair offers [40]. It is also consistent with the findings that skin conductance (emotional arousal) physiological measures were higher for unfair than fair offers [43].

The current model of emotion also complements Solomon's model of opponent-process theory of motivation in the temporal dynamics of affect [56], in which emotion is described as the result of the opposing hedonic-affective arousal and the slave processes. The driving force for the affective response is a result of the similar opposing pleasure-aversion processes in Pavlovian conditioning [57]. Although the emotional models are similar in terms of the opponent processes that differentiate the happy and unhappy emotions, which leads to pleasure-seeking and aversion-avoidance behaviors, the difference is that the disparity signal in gain/loss in our emotional model is the stimulus that elicits the emotional response. Whereas the pleasure-seeking/aversion-avoidance behavior is not a stimulus, but rather, it is a response to the emotion. The pleasure/pain is a response to the gain/loss signal; but the gain/loss cannot be a response to pleasure/pain. Thus, the assessment of the amount of gain/loss by an animal or human is crucial prior to any response to pleasure/pain or pleasure-seeking/aversion-avoidance behavior. This sets our emotional model apart from other emotional models. Because the amount of gain/loss is quantifiable (and can be measured objectively), our emotional model is also quantifiable based on the response to the quantifiable gain/loss signal. On the other hand, because the stimuli of other emotional models are often subjective and unquantifiable with objective measures, it presents difficulties in experimental validation of the models.

This provides the first of a series of experiments to validate the emotion model, while further experiments will be needed to delineate the causal relationships between the emotional responses, decisions, fairness, gain/loss signals, expectancy, reward prediction and loss aversion. The relationship between emotion and decision is addressed in the companion paper [58].

\section{Summary}

This study demonstrated that emotion responses can be elicited by using the disparity signals as the stimuli in the classical UG paradigm. It revealed that the emotional responses are proportional to the offer-ratios graphically by the emotion stimulus-response function. It quantified the sense of objective fairness in equality can override the sense of subjective fairness in hyper-fairness, even though a person can potentially gain more. It also quantified that the threshold for happy emotion is not set at the absolute equity of $\$ 5: \$ 5$ even-split offer-ratio. Instead, the threshold for happy emotion is set at a much lower unfair-ratio (inequity) - at the $\$ 2: \$ 8$ offer-ratio — than the absolute equity $\$ 5: \$ 5$ offer-ratio. This suggests that humans are rather lenient to inequity without getting angry.

Furthermore, the sensitivity to happiness also changes depending on whether the offers are unfair or hyper-fair. This is quantified graphically by the change in the slope of the stimulus-response function, with a shallower slope for the hyper-fair offers than the unfair offers. The emotional baseline is also changed by the fairness perception (shifting from a negative emotional intensity for unfair-offers to a positive emotional intensity for hyper-fair offers. This is quantified by the change in the $y$-intercept, shifting from a negative emotional baseline for the unfair offers to a positive emotional baseline for the hyper-fair offers.

\section{Conclusion}

The experimental evidence using the UG paradigm shows that the emotional response can be quantified graphically by the stimulus-response function. The happy emotional intensity is shown to be proportional to the offer-ratio in the UG paradigm. The proportionality relationship can be changed by the fairness perception. The emotional baseline is shown quantitatively to shift from a negative emotional intensity for the unfair offers to a positive emotional intensity for the hyper-fair offers. The emotional sensitivity is also shown quantitatively to change from a highly sensitive emotion for the unfair offers to a less sensitive emotion for the hyper-fair offers. This is represented by a change from a steeper slope to a shallower slope in the stimulus-response function. These quantitative results are consistent with the intuitive qualitative assessment of emotional responses, validating the proposed theoretical emotional model that the intensity of emotional response is proportional to the magnitude of the perceived gain, which can be biased by the fairness perception.

\section{Acknowledgements}

I greatly appreciate Ms. Krista Smith for the helpful suggestions, and for proofreading the manuscript. 


\section{References}

[1] D. Tam, "EMOTION-I model: A biologically-based theoretical framework for deriving emotional context of sensation in autonomous control systems," Open Cybern Sys $J$, vol. 1, pp. 28-46, 2007.

[2] D. Tam, "EMOTION-II model: A theoretical framework for happy emotion as a self-assessment measure indicating the degree-of-fit (congruency) between the expectancy in subjective and objective realities in autonomous control systems," Open Cybern Sys J, vol. 1, pp. 47-60, 2007.

[3] D. N. Tam, "Computation in emotional processing: quantitative confirmation of proportionality hypothesis for angry unhappy emotional intensity to perceived loss," Cognitive Computation, vol. 3, pp. 394-415, 18 July 2011 2011.

[4] D. Tam, "A theoretical model of emotion processing for optimizing the cost function of discrepancy errors between wants and gets," BMC Neuroscience, vol. 10, p. P11, Jul 13 2009.

[5] F. Schneider, R. C. Gur, R. E. Gur, and L. R. Muenz, "Standardized mood induction with happy and sad facial expressions," Psychiatry Res, vol. 51, pp. 19-31, Jan 1994.

[6] R. J. Davidson, D. Mednick, E. Moss, C. Saron, and C. E. Schaffer, "Ratings of emotion in faces are influenced by the visual field to which stimuli are presented," Brain Cogn, vol. 6, pp. 403-11, Oct 1987.

[7] B. Derntl, E. M. Seidel, F. Schneider, and U. Habel, "How specific are emotional deficits? A comparison of empathic abilities in schizophrenia, bipolar and depressed patients," Schizophr Res, vol. 142, pp. 58-64, Dec 2012.

[8] J. Jaeger, J. C. Borod, and E. Peselow, "Facial expression of positive and negative emotions in patients with unipolar depression," J Affect Disord, vol. 11, pp. 43-50, Jul-Aug 1986.

[9] S. Srivastava, H. O. Sharma, and M. K. Mandal, "Mood induction with facial expressions of emotion in patients with generalized anxiety disorder," Depress Anxiety, vol. 18, pp. $144-8,2003$.

[10] V. I. Muller, T. S. Kellermann, S. C. Seligman, B. I. Turetsky, and S. B. Eickhoff, "Modulation of affective face processing deficits in schizophrenia by congruent emotional sounds," Soc Cogn Affect Neurosci, Oct 142012.

[11] B. Lorey, M. Kaletsch, S. Pilgramm, M. Bischoff, S. Kindermann, I. Sauerbier, et al., "Confidence in emotion perception in point-light displays varies with the ability to perceive own emotions," PLoS One, vol. 7, p. e42169, 2012.

[12] P. D. Parker, K. M. Prkachin, and G. C. Prkachin, "Processing of facial expressions of negative emotion in alexithymia: the influence of temporal constraint," $J$ Pers, vol. 73, pp. 1087-107, Aug 2005.

[13] I. Laeger, C. Dobel, U. Dannlowski, H. Kugel, D. Grotegerd, J. Kissler, et al., "Amygdala responsiveness to emotional words is modulated by subclinical anxiety and depression," Behav Brain Res, vol. 233, pp. 508-16, Aug 12012.

[14] M. J. van Tol, L. R. Demenescu, N. J. van der Wee, R.
Kortekaas, M. A. N. Marjan, J. A. Boer, et al., "Functional magnetic resonance imaging correlates of emotional word encoding and recognition in depression and anxiety disorders," Biol Psychiatry, vol. 71, pp. 593-602, Apr 12012.

[15] J. von Neumann, O. Morgenstern, and A. Rubinstein, Theory of games and economic behavior. Princeton, NJ: Princeton University Press, 1953.

[16] J. H. Kagel and A. E. Roth, The handbook of experimental economics: PRINCETON University Press, 1995.

[17] D. A. Braun, P. A. Ortega, and D. M. Wolpert, "Nash equilibria in multi-agent motor interactions," PLoS Comput Biol, vol. 5, p. e1000468, Aug 2009.

[18] K. Sigmund, C. Hauert, and M. A. Nowak, "Reward and punishment," Proc Natl Acad Sci US A, vol. 98, pp. 10757-10762, Sep 112001.

[19] A. Bechara, "The role of emotion in decision-making: evidence from neurological patients with orbitofrontal damage," Brain Cogn, vol. 55, pp. 30-40, Jun 2004.

[20] C. Civai, C. Corradi-Dell'Acqua, M. Gamer, and R. I. Rumiati, "Are irrational reactions to unfairness truly emotionally-driven? Dissociated behavioural and emotional responses in the Ultimatum Game task," Cognition, vol. 114, pp. 89-95, Jan 2010.

[21] J. D. Greene, L. E. Nystrom, A. D. Engell, J. M. Darley, and J. D. Cohen, "The neural bases of cognitive conflict and control in moral judgment," Neuron, vol. 44, pp. 389-400, Oct 142004.

[22] K. M. Harle and A. G. Sanfey, "Incidental sadness biases social economic decisions in the Ultimatum Game," Emotion, vol. 7, pp. 876-881, Nov 2007.

[23] S. M. McClure, D. I. Laibson, G. Loewenstein, and J. D. Cohen, "Separate neural systems value immediate and delayed monetary rewards," Science, vol. 306, pp. 503-7, Oct 152004.

[24] E. K. Miller and J. D. Cohen, "An integrative theory of prefrontal cortex function," Annu Rev Neurosci, vol. 24, pp. 167-202, 2001.

[25] G. J. Quirk and J. S. Beer, "Prefrontal involvement in the regulation of emotion: convergence of rat and human studies," Curr Opin Neurobiol, vol. 16, pp. 723-7, Dec 2006.

[26] E. T. Rolls, "Brain mechanisms of emotion and decision-making," Int Congress Series, vol. 1291, pp. 3-13, 2006.

[27] A. G. Sanfey, G. Loewenstein, S. M. McClure, and J. D. Cohen, "Neuroeconomics: cross-currents in research on decision-making," Trends Cogn Sci, vol. 10, pp. 108-16, Mar 2006.

[28] A. G. Sanfey, J. K. Rilling, J. A. Aronson, L. E. Nystrom, and J. D. Cohen, "The neural basis of economic decision-making in the Ultimatum Game," Science, vol. 300, pp. 1755-1758, Jun 132003.

[29] J. K. Rilling, A. G. Sanfey, J. A. Aronson, L. E. Nystrom, and J. D. Cohen, "The neural correlates of theory of mind within interpersonal interactions," Neuroimage, vol. 22, pp. 1694-703, Aug 2004. 
[30] P. Smith and A. Silberberg, "Rational maximizing by humans (Homo sapiens) in an ultimatum game," Anim Cogn, vol. 13, pp. 671-7, Jul 2010.

[31] T. Yamagishi, Y. Horita, H. Takagishi, M. Shinada, S. Tanida, and K. S. Cook, "The private rejection of unfair offers and emotional commitment," Proc Natl Acad Sci U S A, vol. 106, pp. 11520-11523, Jul 142009.

[32] S. S. Komorita, "Attitude content, intensity, and the neutral point on a Likert scale," J Soc Psychol, vol. 61, pp. 327-34, Dec 1963.

[33] T. C. Burnham, "High-testosterone men reject low ultimatum game offers," Proc Biol Sci, vol. 274, pp. 2327-30, Sep 222007.

[34] C. Eisenegger, M. Naef, R. Snozzi, M. Heinrichs, and E. Fehr, "Prejudice and truth about the effect of testosterone on human bargaining behaviour," Nature, vol. 463, pp. 356-9, Jan 212010 .

[35] B. Güroğlu, W. van den Bos, and E. A. Crone, "Fairness considerations: increasing understanding of intentionality during adolescence," J Exp Child Psychol, vol. 104, pp. 398-409, Dec 2009.

[36] B. Güroğlu, W. van den Bos, S. A. Rombouts, and E. A. Crone, "Unfair? It depends: neural correlates of fairness in social context," Soc Cogn Affect Neurosci, vol. 5, pp. 414-423, Dec 2010.

[37] M. Koenigs and D. Tranel, "Irrational economic decision-making after ventromedial prefrontal damage: evidence from the Ultimatum Game," J Neurosci, vol. 27, pp. 951-6, Jan 242007.

[38] M. A. Nowak, K. M. Page, and K. Sigmund, "Fairness versus reason in the ultimatum game," Science, vol. 289, pp. 1773-1775, Sep 82000

[39] M. M. Pillutla and J. K. Murnighan, "Unfairness, anger, and spite: Emotional rejections of ultimatum offers," Organizational Behavior and Human Decision Processes, vol. 68, pp. 208-224, 12// 1996.

[40] G. Tabibnia, A. B. Satpute, and M. D. Lieberman, "The sunny side of fairness: preference for fairness activates reward circuitry (and disregarding unfairness activates self-control circuitry)," Psychol Sci, vol. 19, pp. 339-347, Apr 2008.

[41] D. N. Tam, "Computation in emotional processing: quantitative confirmation of proportionality hypothesis for angry unhappy emotional intensity to perceived loss," Cogn Comput, vol. 3, pp. 394-415, 2011/06/01 2011.

[42] N. D. Tam, "Assessing fairness bias using a relativistic fairness-equity model: theoretical derivation," submitted.

[43] M. van't Wout, R. S. Kahn, A. G. Sanfey, and A. Aleman, "Affective state and decision-making in the Ultimatum Game," Exp Brain Res, vol. 169, pp. 564-8, Mar 2006.
[44] P. J. Zak, R. Kurzban, S. Ahmadi, R. S. Swerdloff, J. Park, L Efremidze, et al., "Testosterone administration decreases generosity in the ultimatum game," PLoS One, vol. 4, p. e8330, 2009.

[45] P. J. Zak, A. A. Stanton, and S. Ahmadi, "Oxytocin increases generosity in humans," PLoS One, vol. 2, p. e1128, 2007.

[46] D. Tam, "Variables governing emotion and decision-making: human objectivity underlying its subjective perception," BMC Neuroscience, vol. 11, p. P96, Jul 202010.

[47] E. Xiao and D. Houser, "Emotion expression in human punishment behavior," Proc Natl Acad Sci U S A, vol. 102, pp. 7398-401, May 172005.

[48] D. N. Tam, "Contributing factors in judgment of fairness by monetary value," in BMC Neuroscience, 2011, p. P329.

[49] D. N. Tam, "Quantification of fairness bias by a Fairness-Equity Model," BMC Neuroscience, vol. 12, p. P327, 2011.

[50] E. C. Seip, W. W. van Dijk, and M. Rotteveel, "On hotheads and Dirty Harries: the primacy of anger in altruistic punishment," Ann N Y Acad Sci, vol. 1167, pp. 190-196, Jun 2009.

[51] E. A. Murray and A. Izquierdo, "Orbitofrontal cortex and amygdala contributions to affect and action in primates," Ann N Y Acad Sci, vol. 1121, pp. 273-96, Dec 2007.

[52] S. E. Morrison and C. D. Salzman, "Re-valuing the amygdala," Curr Opin Neurobiol, vol. 20, pp. 221-30, Apr 2010.

[53] J. P. O'Doherty, "Lights, camembert, action! The role of human orbitofrontal cortex in encoding stimuli, rewards, and choices," Ann N Y Acad Sci, vol. 1121, pp. 254-72, Dec 2007.

[54] E. A. Murray and S. P. Wise, "Interactions between orbital prefrontal cortex and amygdala: advanced cognition, learned responses and instinctive behaviors," Curr Opin Neurobiol, vol. 20, pp. 212-20, Apr 2010.

[55] S. M. Tom, C. R. Fox, C. Trepel, and R. A. Poldrack, "The neural basis of loss aversion in decision-making under risk," Science, vol. 315, pp. 515-8, Jan 262007.

[56] R. L. Solomon and J. D. Corbit, "An opponent-process theory of motivation. I. Temporal dynamics of affect," Psychol review, vol. 81, pp. 119-45, Mar 1974.

[57] R. A. Rescorla and R. L. Solomon, "Two-process learning theory: Relationships between Pavlovian conditioning and instrumental learning," Psychol Rev, vol. 74, pp. 151-82, May 1967.

[58] Psychology and Behavioral Sciences 2014; 3(2): 68-74. 